\title{
Incidence of Bacteremia After Endoscopic Variceal Ligation of Oesophageal Varices in Cirrhotic Patients
}

\author{
Alam MS ${ }^{1}$, Lama $\mathrm{TK}^{2}$, Alam $\mathrm{AKMK}^{3}$
}

\begin{abstract}
Bacteremia and infectious sequelea are frequent complications after sclerotherapy. As cirrhotic patients are immune deficient, bacteremia occurring as a consequence of endoscopic variceal ligation (EVL) may develop systemic infection. The aim of the study was to see the incidence of bacteremia in cirrhotic patients after EVL.
\end{abstract}

In this study 91 cirrhotic patients with grade II-III oesophageal varices were enrolled and 100 EVL sessions were done. Patients receiving antibiotics, having evidence of infection were excluded. After the completion of EVL, blood samples for culture and sensitivity were collected at 5 minute and after 30minute and subculture done in Blood Agar and MacConkey Agar media. We followed the patients for a week.

Of the one hundred cases there were 30 subjects in Child A, 48 in Child B and 13 in Child C class. Four patients (4\%) had positive blood culture after EVL, three of Child A class and one of Child B class and non in Child C class. All three patients of Child $A$ had culture positive in their 30minute sample and not in 5 minute sample. The Child B patient had culture positive in his 5minute sample and not in 30minute sample. The bacterium isolated was Coagulase negative Staphylococcus, which we considered it as contaminant. None of these patients developed any infectious sequelea.

The incidence of bacteremia after EVL is yet to be ascertained. Post EVL antibiotic prophylaxis is not recommended.

\section{Introduction}

Bacterial infections are one of the important causes of death in cirrhotic patient which accounts for $38 \%$, either directly or indirectly ${ }^{1,2}$. In hospitalized cirrhotic patients, the incidence of bacterial infections is 32 to $34 \% \%^{3,4}$ and in those

1. Corresponding Author:

Md. Shahinul Alam MBBS, FCPS, MD

Associate Professor, Department of Hepatology

Bangabandhu Sheikh Mujib Medical University, Bangladesh

E-mail: shahinul67@yahoo.com

2. Thupten K Lama MBBS

Resident, Department of Hepatology

Bangabandhu Sheikh Mujib Medical University, Bangladesh

3. AKM Khorshed Alam MBBS, FCPS, MHPE, FRCP

Professor and Chairman, Department of Hepatology

Bangabandhu Sheikh Mujib Medical University, Bangladesh with gastrointestinal (GI) hemorrhage is about $45 \%$. This infection rate is much higher than the 5 to $7 \%$ infection rate reported in the general population of hospitalized patients. This increased rate is mostly due to an associated immunocompromised state resulting in deficient defense mechanism ${ }^{2}$. These cirrhotic patients also have a higher relative risk of dying from sepsis than non-cirrhotic patients ${ }^{6}$. The most common bacterial infections are spontaneous bacterial peritonitis (SBP) $(25 \%)$, urinary tract infections (UTI) (20\%), pneumonia (15\%) and bacteremia $(12 \%)^{4}$.

Transient bacteremia is frequent event occurring after upper gastrointestinal endoscopy with a reported incidence of $4 \%$ in one study ${ }^{7}$. Bacterial translocation of endogenous microbial flora into the bloodstream may occur during an endoscopy because of mucosal trauma related to the procedure.

EVL is now the treatment of choice over sclerotherapy for bleeding and nonbleeding varices. Various prospective studies have been conducted to evaluate the frequency of occurrence of post EVL bacteremia with incidence ranging from $0 \%$ to $25 \%{ }^{8-14}$.

Most bacteremia do not cause symptoms and have no clinical significance. However in cirrhotic patient (who are immunodeficient) it is of major clinical importance ${ }^{15}$.

In view of this existing immunologic deficit and bypass in the reticuloendothelial system resulting from collateral circulation of portal system in cirrhotics, a transient bacteremia may become permanent. This would favour the seeding of bacteria to distant sites with development of infection ${ }^{15}$.

\section{Materials and Methods}

This observational prospective study was carried out in the period from September 2010 to September 2011 in the department of Hepatology, Bangabandhu Sheikh Mujib Medical University, Dhaka, Bangladesh. A total of 144 EVL sessions were done during this period. The inclusion criteria included all cirrhotic patients undergoing EVL. Exclusion criteria were use of antibiotics within 14 days before endoscopy, any sign of infection evidenced by fever (axillary temperature higher than or equal to $1000 \mathrm{~F}$ ) within 48hours before endoscopy, use of any indwelling catheter or central venous line, haemo-dynamically unstable, current use of corticosteroids or immunosuppressant and age less than 15 or more than 60 years.

After screening the patients according to exclusion criteria, 91 patients were enrolled in the study and $100 \mathrm{EVL}$ sessions 
were done. The cirrhotic patients were classified according to Child Pugh criteria. Upper GI endoscopy was performed using the endoscopic device Olympus EXERA II. Among the 44 excluded cirrhotic patients, 23 did not consent to study and remaining 21 were on antibiotic therapy. A total of 16 Child C patients and 4 Child B \& 1 Child A patients were excluded.

Number and grade of oesophageal varices with or without red sign were noted during endosocpy. Using multiband ligator (V GRIPP, Indus Medical Instruments private limited) containing 6 bands released by V GRIPP release handle, EVL was done in all enrolled patients.

Venous blood was collected at 5minute and after 30minute of completion of procedure. $10 \mathrm{ml}$ each of blood samples were drawn from different sites after cleansing the skin with alcohol and sent in culture bottle (containing brain heart infusion broth) for aerobic culture and sensitivity testing. The samples were incubated at $37^{\circ} \mathrm{C}$ for overnight after which subculture were done in MacConkey agar and Blood agar media. A total of 200 blood samples were collected in culture bottle and incubated. The patients were followed for a week to detect any sign of infection.

Informed consent was obtained from the patients or their relatives before being included in the study, which was approved by the Ethical Committee of our Institution.

\section{Results}

In our study a total of 91 patients of cirrhosis of liver with oesophageal varices Grade II-III were enrolled and 100 EVL sessions were done. The mean age of the study group was $41.77 \pm 12.54$ years. There was male 90 (90\%) predominance in this study (table 1).

Table 1: Patient demographic and baseline characteristics.

\begin{tabular}{|c|c|c|c|c|c|}
\hline \multicolumn{2}{|c|}{ Variable } & \multirow{2}{*}{$\begin{array}{c}\begin{array}{c}\text { Child A } \\
\mathbf{n}=\mathbf{3 9}\end{array} \\
19\end{array}$} & \multirow{2}{*}{$\begin{array}{c}\begin{array}{c}\text { Child B } \\
\mathbf{n}=\mathbf{4 8}\end{array} \\
8\end{array}$} & \multirow{2}{*}{$\begin{array}{c}\begin{array}{c}\text { Child C } \\
\mathbf{n}=\mathbf{1 3}\end{array} \\
2\end{array}$} & \multirow{2}{*}{$\begin{array}{c}\begin{array}{c}\text { Total } \\
\mathbf{n}=100\end{array} \\
29\end{array}$} \\
\hline Age & $16-30$ & & & & \\
\hline & $31-45$ & 10 & 18 & 4 & 32 \\
\hline & $46-60$ & 10 & 22 & 7 & 39 \\
\hline \multirow[t]{2}{*}{ Sex } & Male & 36 & 41 & 13 & 90 \\
\hline & Female & 3 & 7 & 0 & 10 \\
\hline \multicolumn{2}{|c|}{ AetiologyHBV } & 24 & 39 & 9 & 72 \\
\hline \multicolumn{2}{|r|}{$\mathrm{HCV}$} & 4 & 6 & 0 & 10 \\
\hline \multirow{2}{*}{\multicolumn{2}{|c|}{$\begin{array}{l}\text { NBNC } \\
\mathrm{HBV}, \mathrm{HCC}\end{array}$}} & 11 & 2 & 2 & 15 \\
\hline & & $\mathrm{C}$ & 0 & 2 & 2 \\
\hline \multirow{2}{*}{\multicolumn{2}{|c|}{ Treatment }} & 0 & 1 & 0 & 1 \\
\hline & & 9 & 15 & 8 & 32 \\
\hline \multicolumn{2}{|c|}{$\begin{array}{l}\text { Pri Prophylaxis } \\
\text { Sec Prophylaxis }\end{array}$} & 30 & 33 & 5 & 68 \\
\hline
\end{tabular}

*Pri - Primary prophylaxis, ${ }^{*}$ Sec - Secondary prophylaxis

Thirty nine (39\%) patients were of Child A class, 48 (48\%) were of Child B class and $13(13 \%)$ were of Child C class. Hepatitis B infection was the most common cause $72(72 \%)$ followed by non B non C infection 15 (15\%), Hepatitis C infection $10(10 \%)$ and remaining $2(2 \%)$ had concomitant HCC. One was a case of ALD. Sixty eight percent of subjects underwent EVL for secondary prophylaxis and $32 \%$ for primary prophylaxis.
In our study four patients (4\%) had positive blood culture after EVL (table 2), three in Child A class and one in Child $B$ class. None of the patients with bacteremia developed any infection. The isolated organism was Coagulase negative Staphylococcus in all four patients.

Table 2: Positive blood culture in different child pugh class

\begin{tabular}{|lcccc|}
\hline Culture & Child A & Child B & Child C & Total \\
\hline 5min & & & & \\
Negative & 39 & 47 & 13 & 99 \\
Positive & 0 & 1 & 0 & 1 \\
Organism & - & CNS $^{*}$ & - & - \\
30min & & & & \\
Negative & 36 & 48 & 13 & 97 \\
Positive & 3 & 0 & 0 & 3 \\
Organism & CNS $^{*}$ & - & - & - \\
\hline
\end{tabular}

*CNS- Coagulase negative staphylococcus

All three patients of Child A had positive culture in 30 minute sample only. The Child B patient had positive culture at 5minute sample only. The number of band applied ranged from 3 to 6 .

\section{Discussion}

In this study HBV infection was most common cause of cirrhosis. HBV infection was the most common cause of cirrhosis seen in study conducted by Afroz. in same department in $2007^{16}$. This compared to other countries like India and Brazil, alcohol was a major cause of cirrhosis ${ }^{8,9}$.

In this study positive blood culture was detected in 4 patients (4\%) after the procedure. This rate of $4 \%$ bacteremia following EVL is in line with other published data of $1 \%$ to $25 \%$ (mean rate of $8.8 \%$ ).

The organism isolated in this study was Coagulase negative Staphylococcus in each of the 4 patients. Other studies have isolated different organisms. Kulkarni also isolated the same organism in addition to Enterobactor and Klebsiella ${ }^{8}$. Similarly Tseng reported Staphylococcus epidermidis which is also a Coagulase negative Staphylococcus after the $\mathrm{EVL}^{10}$.

This bacterium is generally interpreted as contamination by most laboratories. The mode of entry in these subjects may have been by the endoscope or contamination during the sample collection as this is naturally found in skin of healthy adults. The bacteremia in one subject can be described as transient as organism was isolated in 5minute sample and not in the 30minute sample. In the remaining three Child A subjects the bacteremia cannot be defined as transient as there was no bacteremia in the first sample but only in the second sample.

It has been suggested that liver dysfunction is an independent risk factor for bacteremia following EVL. But some authors do not support this. This study also does not support this issue as because all bacteremia occurred in Child A and Child B patients and non in Child C. Again 
this scenario does not give the whole picture since Child C patients were very less enrolled and many excluded were on antibiotics. Study by Maulaz also shared this reason for not supporting the view of bacteremia being common in patients with severe liver dysfunction ${ }^{9}$.

The relationship of bacteremia with number of bands applied is not known. In our study the average band applied ranged from 3 to 6 . Rhor reported 3 bands application in their two cases of bacteremia ${ }^{11}$. Similarly Maulaz also reports 3 bands application in their single bacteremic case ${ }^{9}$. The authors in remaining studies have not mention about the band numbers.

None of these four subjects in this study developed any infectious sequelea. Regarding other complications, chest pain, retrosternal pain and dysphagia was common in almost all subjects undergoing EVL. Search of previous literature shows incidence of infectious sequelea being more common after injection sclerotherapy than after EVL $(18 \% \text { vs } 1.8 \%)^{12}$. In our study there was no incidence of infectious sequelea seen after EVL in the four patients with bacteremia. Various studies have reported bacterial peritonitis after EVL. Lo GH reported SBP being the most common of infectious sequelea though the bacteria causing it are not mentioned (1994) ${ }^{12}$. Lin also found no SBP development in subjects with bacteremia undergoing EVL though two subjects developed peritonitis with E. coli but with no bacteremia $(2000)^{13}$. So that was not conclusive of post EVL peritonitis. These findings suggest that infectious sequelea is very much less common after EVL.

In conclusion, the rate of bacteremia after EVL is yet to be ascertained and so post EVL antibiotic prophylaxis is not recommended.

\section{References}

1. Caly WR, Strauss E. A prospective study of bacterial infections in patients with cirrhosis. J Hepatol 1993;18:353-8.

2. Rosa H, Silvério AO, Perini RF, Arruda CB. Bacterial infection in cirrhotic patients and its relationship with alcohol. Am J Gastroenterol 2000;95:1290 -3.

3. Borzio M, Salerno F, Piantoni L, Cazzaniga, M, Angeli P, Bissoli F et al. Bacterial infection in patients with advanced cirrhosis: a multicentre prospective study. Dig Liver Dis 2001;33:41-48

4. Fernandez J, Navasa M, Gomez J, Colmenero J, Vila $\mathrm{J}$, Arroyo V, et al. Bacterial infections in cirrhosis: epidemiological changes with invasive procedures and norfloxacin prophylaxis. Hepatology 2002;35:140-148
5. Bernard B, Grange JD, Khac EN, Amiot X, Opolon P, Poynard T. Antibiotic prophylaxis for the prevention of bacterial infections in cirrhotic patients with gastrointestinal bleeding: a metaanalysis. Hepatology 1999;29:1655-1661

6. Foreman MG, Mannino DM, Moss M. Cirrhosis as a risk factor for sepsis and death: analysis of the national hospital discharge survey. Chest 2003;124:1016-1020

7. Botomon VA, Surawicz CM. Bacteremia with gastrointestinal endoscopic procedure. Gastrointest Endosc 1986;32:342-6

8. Kulkarni SG, Parikh SS, Dhawan PS, Chachad H, Jambavalikar MB, Koppikar GV et al. High frequency of bacteremia with endoscopic treatment of esophageal varices in advanced cirrhosis. Indian J Gastroenterol 199918:143-5

9. Maulaz EB, Mattos AA, Pereira-Lima J, Dietz J. Bacteremia in cirrhotic patients submitted to endoscopic band ligation of esophageal varices. Arq Gastroenterol 2003;40:166-72

10. Tseng CC, Green RM, Burke SK, Connors PJ, Carr LDL. Bacteremia after endoscopic band ligation of esophageal varices. Gastrointest Endosc 1992;38:336-7

11. Rohr MRS, Siqueira ES, Brant CQ, Morais M, Libera EDL, Castro RR, Ferrari AP. Prospective study of bacteremia rate after elastic band ligation and sclerotherapy of esophageal varices in patients with hepatosplenic schistosomiasis. Gastrointest Endosc 1997;46:321-3

12. Lo GH, Lai KH, Shen MT, Chang CF. A comparison of the incidence of transient bacteremia and infectious sequelae after sclerotherapy and rubber band ligation of bleeding esophageal varices. Gastrointest Endosc 1994;40:675-9

13. Lin OS, Wu SS, Chen YY, Tsai YT, Lin HC, Lu RH et al. Bacterial peritonitis after elective endoscopic variceal ligation: a prospective study. Am J Gastroenterol 2000;95:214-17

14. Berner JS, Gaing AA, Sharma R, Almenoff PL, Muhlfelder T, Korsten MA. Sequelae after esophageal variceal ligation and sclerotherapy: a prospective randomized study. Am J Gastroenterol 1994;89:852-8

15. Rimola A, Soto R, Bory F, Arroyo V, Piera C, Rodes, J. Reticuloendothelial system phagocytic activity in cirrhosis and its relation to bacterial infections and prognosis. Hepatology 1984;4:53-8

16. Afroz S, Mahtab MA, Rahman S, Khan M. Hepatitis $\mathrm{B}$ is the leading cause of cirrhosis of liver in Bangladesh. Hep Intl 2007;1:3-242 